\title{
LETTER
}

\section{Number needed to treat for subglottic secretion drainage technology as a ventilator-associated pneumonia prevention strategy}

\author{
Scott D Kelley* \\ See related letter by Wyncoll and Camporota, http://ccforum.com/content/16/3/430
}

\begin{abstract}
The number needed to treat can be calculated for ventilator-associated pneumonia reduction strategies such as subglottic secretion drainage technology based on previous work establishing its relative risk reduction. Assuming an incidence of $4 \%$, employing subglottic secretion drainage in 33 patients will prevent one case of ventilator-associated pneumonia, and thus potentially 4 cases annually in an average hospital in the United States. With a previously described limit of $£ 300$ (\$470 USD) additional cost per 10 days of ventilation as a threshold of investment for technologies to reduce ventilator-associated pneumonia, subglottic secretion drainage technology is both clinically and cost effective.
\end{abstract}

Wyncoll and Camporota's correspondence [1] provides a valuable tool allowing clinicians the ability to assess the number needed to treat (NNT) and cost-effectiveness of various ventilator-associated pneumonia (VAP) prevention strategies. Subglottic secretion drainage (SSD) technology is one VAP-reduction strategy for which NNT can easily be calculated based on its established relative risk reduction.

Smulders and colleagues [2] conducted a randomized clinical trial in 150 patients receiving mechanical ventilation and found that intermittent SSD reduced the risk of VAP by $75 \%(P=0.014)$. Utilizing Wyncoll and Camporota's table, and assuming a VAP incidence of $4 \%$, utilizing SSD in 33 patients will prevent one episode of

*Correspondence: scott.kelley@covidien.com

Respiratory and Monitoring Solutions, Covidien, 6135 Gunbarrel Dr., Boulder, CO 80301, USA
VAP. In the United States, the average hospital has approximately 131 patients per year who require mechanical ventilation $>96$ hours (unpublished observations). Utilizing SSD in these average hospitals would likely prevent four cases of VAP per year. In addition, SSD is a cost-effective intervention. At the assumed VAP rate of $4 \%$, Wyncoll and Camporota determined an upper threshold of $£ 300$ (\$470 USD) additional cost per 10 days of ventilation to be a cost-effective investment in technologies that reduce VAP by up to $75 \%$. Costs of SSD technology interventions are beneath this threshold.

Wyncoll and Camporota's framework illustrates that use of SSD is both a clinically and cost-effective strategy to reduce VAP. The NNT demonstrates that average hospitals will observe and benefit from these strategies. SSD technologies warrant further adoption and appropriate utilization.

\section{Abbreviations \\ NNT, number needed to treat; SSD, subglottic secretion drainage; VAP, ventilator-associated pneumonia.}

\section{Competing interests}

SK is an employee of Covidien, and receives salary and equity compensation.

\section{Acknowledgements}

SK would like to thank Mary Erslon, RN, MSN, MBA (Covidien) for writing assistance.

\section{Published: 4 September 2012}

\section{References}

1. Wyncoll D, Camporota L: Number needed to treat and cost-effectiveness in the prevention of ventilator-associated pneumonia. Crit Care 2012, 16:430.

2. Smulders $\mathrm{K}$, can der Hoeven $\mathrm{H}$, Weers-Pothoff I, Vandenbroucke-Grauls C:

A randomized clinical trial of intermittent subglottic secretion drainage in patients receiving mechanical ventilation. Chest 2002, 121:858-862.

\section{doi:10.1186/cc11464}

Cite this article as: Kelley SD: Number needed to treat for subglottic secretion drainage technology as a ventilator-associated pneumonia prevention strategy. Critical Care 2012, 16:446. 